\title{
Un instrumento innovador para defragmentar a propiedade? A permuta entre múltiples participantes e a percepción dos gandeiros
}

\author{
Recibido: 3 outubro 2017 / Aceptado: 15 novembro 2017
}

\begin{abstract}
Resumo Neste traballo explórase a viabilidade dun proceso de permuta de uso das parcelas entre múltiples participantes como ferramenta para reducir a fragmentación do uso da terra e mellorar a estrutura parcelaria das explotacións. Trátase de identificar os criterios e variables máis relevantes a hora de realizar un proceso deste tipo, desde a perspectiva das explotacións de gando vacún, co obxectivo adicional de que o coñecemento xerado sirva de base para o deseño de ferramentas informáticas de apoio. A través da realización de entrevistas e a análise cualitativa dos resultados obtidos, quedou patente que a marxe de mellora da base territorial das explotacións existe e que a práctica da permuta non é totalmente allea ao sector. $\mathrm{O}$ traballo permitiu explorar cales serían as principais vantaxes e inconvenientes dun proceso destas características desde a perspectiva dos seus potenciais beneficiarios.
\end{abstract}

Palabras clave Permuta de uso, fragmentación da propiedade, estrutura das explotacións.

An innovative tool to defrag property? Multi-stakeholder parcel exchange in farmers' perspective

Abstract In this work we explore the viability of multistakeholder parcel exchange as a tool to reduce land use fragmentation and improve the structure of farms. We aimed at identifying the criteria and the most relevant variables from the perspective of beef and dairy cattle farmers, hoping that the knowledge generated serves as the basis for the design of information technology-based support tools. Qualitative analysis of information gathered in personal interviews with farmers and local technicians made it clear

Nieves Perez Rodríguez · Eduardo José Corbelle Rico · Francisco José Ónega López

Laboratorio do Territorio, Departamento de Enxeñería Agroforestal, Universidade de Santiago de Compostela. Escola Politécnica Superior de Enxeñería, Benigno Ledo s/n, 27002 - Lugo (España). Email: nieves.perez@usc.es

Tel:+34 982822832 that there is margin for improvement in the current land structure of farms and that farmers are already familiar with parcel exchange (on a one-to-one basis). Our results allowed us to define the main advantages and disadvantages of a process of these characteristics from the perspective of its potential beneficiaries.

Keywords Parcel use exchange, fragmentation of property, structure of farms.

\section{Introdución}

Dende hai décadas, unha elevada fragmentación dos sistemas de propiedade da terra tense considerado un atranco de grande importancia para o desenvolvemento dun sector agrario potente (Binns, 1950). Na bibliografía recente existen numerosos traballos que establecen unha relación entre a fragmentación da propiedade e problemas de xestión das explotacións agrarias (p.ex. Rahman e Rahman, 2009; Manjunatha et al., 2013; Latruffe e Piet, 2014; Orea et al., 2015), ou das explotacións forestais (Rendenieks et al., 2015; Kilgore e Snyder, 2016). En todo caso, este tipo de relacións deben ser examinadas con atención aos casos particulares, dado que tamén existen exemplos nos que non foi posible establecer un vínculo entre unha maior fragmentación e unha menor eficiencia das actividades produtivas (Deininger et al., 2012). Por outra parte, algúns autores teñen resaltado o interese que as propiedades de pequena dimensión -particularmente no caso das propiedades forestais- ten para a conservación da biodiversidade (Schaich e Plieninger, 2013). Como resultado, a afirmación coa que empezamos este texto tende a aceptarse con matices, e enténdese que a redución da fragmentación non ten por que ser un obxectivo das políticas públicas senón en varias situacións particulares: cando esta se propaga ao sistema de uso da terra e resulta nun alto grao de fragmentación da utilización; cando as parcelas resultantes son demasiado pequenas para ser cultivadas de maneira viable; ou cando as parcelas resultantes de cada explotación están moi afastadas entre si (FAO, 2003; Hartvigsen, 2014). 
Agora ben, que entendemos por fragmentación? Seguindo a van Dijk (2003) podemos dicir que existen catro interpretacións posibles do concepto de fragmentación, non necesariamente excluíntes entre si: así, dicimos que o sistema de propiedade está fragmentado cando existe un elevado número de propietarios (o que redunda en pouca superficie media por propietario/a); que exista un elevado número de agricultores ou silvicultores (baixa superficie media por explotación); que non exista solape entre propietarios e agricultores/gandeiros/silvicultores; e que existan moitas máis parcelas que agricultores ou silvicultores (número elevado de parcelas por explotación). $\mathrm{Na}$ práctica, unha área xeográfica pode verse afectada por diferentes combinacións das situacións descritas, con distintas implicacións de tipo ambiental, social e económico, polo que as estratexias privadas e políticas públicas recomendables non sempre son as mesas.

Precisamente, a concentración parcelaria, un proceso no que varias parcelas dun mesmo propietario/a se combinan para formar un número menor de parcelas de maior tamaño (FAO, 2003; Pasakarnis e Maliene, 2010; Pasakarnis et al., 2013; Lisec et al., 2014), é un instrumento de xestión de terras moi utilizado para reducir a fragmentación pero só é útil en casos de elevada fragmentación interna (cando o número de parcelas por propietario, ou por explotación, é moi alto). Polo contrario, non permite reducir a fragmentación noutras situacións, dado que non actúa sobre o número de propietarios ou sobre o número de explotacións.

Pese ao seu carácter central nas políticas públicas de xestión de terras en moitos países, os procesos de concentración parcelaria teñen sido obxecto de importantes críticas polas súas consecuencias de tipo social e ambiental (p.ex. Crecente et al., 2002; Lisec et al., 2014; Kupidura et al., 2014). Trátase, por outra parte, de procesos de elevado custo económico que demandan tempos considerables: Lisec et al. (2014) mencionan 4-5 anos de duración media; Crecente et al. (2002) afirman que poden chegar aos 10 anos. En todo caso, continúa sendo un instrumento que recibe considerable atención en diferentes partes do mundo, como se acredita polo elevado volume de bibliografía recente que se produce sobre o tema (p.ex. Cay e Iscan, 2011; Demetriou et al., 2012; Martínez et al., 2013; Borgwardt et al., 2014; Kupidura et al., 2014; Lisec et al., 2014; Uyan et al., 2015; Haldrup, 2015; Guanghui et al., 2015; Yan et al., 2015; Abubakari et al., 2016; Muchová et al., 2016).

Por outra parte, é un feito documentado na bibliografía que os agricultores e silvicultores tenden a adoptar, por si mesmos, mecanismos que lles permitan reducir a fragmentación das súas propiedades ou das terras que manexan. Normalmente emprégase unha combinación de compra e arrendamento que nalgúns casos permite formar unidades de cultivo suficientemente grandes (Vranken e Swinnen, 2006; Sklenicka et al., 2014). Polo tanto, parece razoable que as políticas públicas de reforma das estruturas agrarias traten de complementar a concentración parcelaria con outros instrumentos máis flexibles e facilmente adaptables a outros contextos, como por exemplo o uso de bancos de terras, ou a permuta voluntaria de parcelas (ou de uso) entre diferentes propietarios ou agricultores/silvicultores, nun esforzo por reorientar a súa actividade cara mecanismos máis próximos á comunidade (van Dijk e Kopeva, 2006; Sikor et al., 2009; Sikor e Müller, 2009).

A permuta de propiedade ou de uso entre múltiples participantes é un sistema empregado en diferentes países, ben en forma de acordos formais ou como acordos temporais de carácter informal. Nalgúns casos, a superficie transferida anualmente a través deste tipo de acordos pode igualar ou mesmo superar á superficie transferida mediante compravenda (man Hui et al., 2014), mesmo coa participación activa do sector público (Segers et al., 2010; Hiironen e Niukkanen, 2014). Trátase dun instrumento de xestión de terras que, ao igual que sucede coa concentración parcelaria, actúa fundamentalmente sobre a fragmentación interna (o número de parcelas do propietario/a ou explotación), e a súa eficacia aumenta co número de participantes e co número de parcelas totais introducidas no proceso. Non obstante, un número elevado de parcelas e participantes supón tamén que o número de posibles combinacións de permuta aumente de maneira moi rápida, e que sexa preciso recorrer a tecnoloxías de computación habituais na resolución de problemas de grande tamaño como, por exemplo, os coñecidos xenericamente como algoritmos heurísticos.

Este tipo de ferramentas teñen sido aplicadas a problemas relacionados coa administración de terras ou o ordenamento territorial como, por exemplo, a asignación de usos do solo (Stewart et al., 2004; Aerts et al., 2005; Kai et al., 2009; Zhang et al., 2010; Suárez et al., 2011; Porta et al., 2013b; Stewart e Janssen, 2014; Liu et al., 2015), a delimitación automática de núcleos de poboación (Porta et al., 2013a), a planificación de espazos verdes (Balling et al., 1999; Xibao et al., 2006; Xin e Zhi-xia, 2008; Vallejo et al., 2015), ou a reasignación de parcelas dentro da concentración parcelaria (Akkus et al., 2012; Demetriou et al., 2012; Uyan et al., 2015).

Se ben as ferramentas técnicas útiles na resolución deste tipo de problemas son relativamente ben coñecidas, a bibliografía é menos abundante no relativo ás actitudes e preferencias dos potenciais beneficiarios da permuta. $O$ obxectivo deste traballo é, precisamente, explorar estes aspectos e, en particular, identificar os criterios e variables de maior relevancia que determinarían a decisión final de participar ou non nun proceso destas características. Para delimitar parcialmente o problema, nesta primeira aproximación exploramos a perspectiva das explotacións especializadas en gando bovino respecto dun hipotético proceso de permuta de uso.

\section{Metodoloxía}

O traballo baseouse na realización dun total de 12 entrevistas semiestruturadas, a técnicos de cooperativas (2) e gandeiros (10). Os individuos foron seleccionados mediante unha técnica de mostraxe non probabilística (mostraxe por conveniencia; Bernal, 2000), apoiada na colaboración de dúas cooperativas que nos facilitaron o 
contacto cos seus asociados. Este tipo de mostraxe non se recomenda para a investigación descritiva ou causal, aínda que pode usarse na investigación exploratoria, como neste caso, a fin de xerar ideas, información ou hipóteses. Deste xeito, trátase dunha técnica de selección común en estudios piloto ou probas de concepto como a que se presenta neste traballo (Malhotra, 1997). As entrevistas tiveron lugar durante os meses de febreiro e abril de 2017, e incluíron a un total de once homes e unha muller con idades comprendidas entre os 30 e os 55 anos. En canto á caracterización profesional, foron entrevistados dous técnicos de cooperativas, un gandeiro de vacún de carne e nove de vacún de leite. Tres contan con activades complementarias (queixería, casa de turismo rural, cebadeiro, e un dos técnicos ademais é gandeiro). No proceso de selección dos entrevistados procurouse cubrir un espectro o máis amplo posible de tipos de manexo (intensivo: animais estabulados, extensivo: animais en pastoreo todo o ano, semi intensivo: parte estabulados e parte en pastoreo), titularidade (Sociedades Agrarias de Transformación, autónomos agrarios, sociedades formadas polo matrimonio), estrutura da base territorial da explotación (explotacións que a tivesen máis concentrada fronte a explotacións con ela máis dispersa), tamaño (entre 50 e 700 animais incluíndo vacas en produción e xovencas), e sexo e idade do titular (Cadro 1). A localización das explotacións visitadas veu condicionada pola distribución da actividade das cooperativas, e centrouse exclusivamente en 8 concellos das provincias de A Coruña e Lugo (Monfero, Aranga, Curtis, Valadouro, Ribadeo, Barreiros, A Pastoriza e Castro de Rei).

Á hora de orientar o contido das entrevistas diferenciouse entre os técnicos das cooperativas e os gandeiros. Dos primeiro buscouse a percepción sobre os problemas de estrutura da base territorial que manexan as explotacións integradas na cooperativa para a que traballan. Dos segundos pretendíase analizar o grao de satisfacción coa base territorial da súa explotación, así como coñecer as dinámicas que ven factibles para mellorar a situación de partida (naqueles casos que pensen que é mellorable).

\section{Resultados}

Segundo se extrae das entrevistas con titulares de explotación, a competencia pola terra entre explotacións veciñas condiciona en boa medida o tamaño medio das parcelas e o seu número e distribución, dado que a terra dispoñible (que procede, no fundamental, de explotacións que deixaron a actividade no pasado recente) é limitada. Cando nunha mesma parroquia coexisten varias explotacións en activo, ou naquelas parroquias nas que a plantación de masas forestais é unha alternativa de uso frecuentemente utilizada polos propietarios, o efecto de competencia é máis acusado. A existencia de procesos de concentración parcelaria no pasado recente así como o modo habitual de reparto das herdanzas poden introducir matices importantes.

O recurso ao arrendamento como vía para incrementar a superficie xestionada é habitual pero non parece xeneralizado, aínda que nalgún caso poida supoñer a maioría da superficie xestionada (é dicir, máis que a superficie que é propiedade dos titulares ou familiares directos). Os prezos dos arrendamentos varían desde a cesión gratuíta aos $800 € /$ ha, e $300 € /$ ha foi indicado como un prezo habitual. É habitual que se trate de acordos verbais, de modo que a práctica totalidade utilizan esta fórmula para algunha das parcelas ou ben a teñen utilizado no pasado recente. Non obstante, tamén existen casos de contratos realizados por escrito, normalmente no caso de explotacións de maiores dimensións e máis profesionalizadas.

\begin{tabular}{|c|c|c|c|c|c|c|}
\hline \multirow[t]{2}{*}{ Explotación } & \multirow[t]{2}{*}{ Manexo } & \multirow[t]{2}{*}{ Titular/es (Idade) } & \multicolumn{2}{|c|}{ Superficie (ha) } & \multirow[t]{2}{*}{ Animais } & \multirow{2}{*}{$\begin{array}{l}\text { Parcela máis } \\
\text { afastada }(\mathrm{km})\end{array}$} \\
\hline & & & En propiedade & Arrendada & & \\
\hline 1 & Intensivo & $45-50$ & 55 & 15 & 180 & 8 \\
\hline 2 & Extensivo & $35-40$ & 27 & 0 & 50 & 1,5 \\
\hline 3 & Extensivo & $50-55$ & 2,5 & 31,5 & 80 & 3 \\
\hline 4 & Intensiva & $40-45$ & 55 & 65 & 220 & 3,5 \\
\hline 5 & Semi-Intensivo & $50-55$ & 20 & 4 & 70 & 2 \\
\hline 6 & Intensivo & $30-35$ & 19 & 7 & 75 & 3 \\
\hline 7 & Intensivo & $50-55$ & 21 & 12 & 70 & 4,5 \\
\hline 8 & Intensivo & $35-40$ & 25 & 55 & 250 & 3 \\
\hline 9 & Intensivo & $35-40$ & 30 & 54 & 230 & 5 \\
\hline 10 & Intensivo & $35-50$ & 145 & 62 & 700 & 20 \\
\hline
\end{tabular}

Cadro 1.- Resumo das características das explotacións visitadas 
A distancia ata a parcela máis afastada da explotación varía moito entre explotacións (atopamos casos entre 2-20 km), pero os casos de parcelas máis afastadas corresponden normalmente a parcelas en propiedade que o titular non desexa deixar de utilizar. Con todo, nun caso particular tratábase dunha parcela arrendada cuxas dimensións e facilidade de acceso compensaban a distancia de desprazamento. Non atopamos casos de parcelas en propiedade que deixaran de ser utilizadas debido a distancia á que se atopan respecto da explotación, e aínda que a maioría dos entrevistados manifestou que non estarían dispostos a desprazarse á igual distancia se o réxime de tenencia fora o arrendamento, tampouco atopamos casos nos os titulares valoraran a posibilidade de permutalas ou arrendalas. Pola contra, se a distancia non semella motivo de abandono da actividade na parcela, a imposibilidade de acceder con maquinaria si foi explicitamente mencionada, especialmente no caso das explotacións que non practican pastoreo (neste caso 7 que teñen manexo intensivo).

Contrariamente á percepción que a miúdo se transmite cara o exterior do sector, a maioría dos entrevistados utilizaron diferentes formas de expresar ("bastante", "moi" ou "razoablemente") o feito de atoparse satisfeitos coa base territorial da súa explotación. Algúns titulares manifestaron explicitamente non estar satisfeitos coa estrutura parcelaria da explotación e as principais causas mencionadas foron a fragmentación en moitas parcelas, a existencia de parcelas afastadas, e tamén a existencia de parcelas con acceso deficiente. En todo caso, todos eles manifestaron estar abertos a explorar vías de mellora, esencialmente pensando en incrementar as dimensións das parcelas, aumentar a proximidade das terras a explotación, ou conseguir mellores accesos, para conseguir así reducir os custos de explotación (tempo de desprazamento, combustible, novas posibilidades de manexo dos animais, redución do perímetro de cerramentos...). As dimensións e a proximidade das parcelas foron situadas sempre en primeiro lugar de importancia (o ideal: parcelas da maior dimensión posible ó lado da explotación), coa calidade dos accesos nun terceiro lugar, pero isto probablemente ten relación co feito de que moitos dos entrevistados están en zonas de concentración parcelaria.

Aproximadamente a metade dos entrevistados indicaron ter feito esforzos para mellorar a situación da estrutura da explotación no pasado. Así, se ben a superficie en réxime de propiedade procede principalmente de herdanzas, atopamos que case tódolos entrevistados mencionaron a realización (por eles mesmos ou polos titulares anteriores) de compras ou permutas orientadas a aumentar a superficie total ou reducir a súa fragmentación. Moitos tamén participan en procesos de concentración parcelaria (ou participaron nalgún recentemente rematado). O principal obstáculo mencionado para utilizar a compra con máis asiduidade foi o do elevado prezo das parcelas (algúns titulares mencionaron 30.000 euros/ha, en parcelas de pequenas dimensións), que converte o arrendamento nunha opción preferible a curto prazo. En xeral, unha combinación de falta de dedicación á busca activa de novas terras, as reticencias dos veciños a arrendar as súas, ou os elevados prezos, nun proceso que parece ter certos elementos de retroalimentación, terían impedido aos titulares mellorar a estrutura das explotacións tanto como lles sería desexable.

Cando se propón a posibilidade de permutar parcelas entre múltiples explotacións, a reacción inicial é moi dispar ("difícil", "complexo","imposible", "factible", "boa idea"...), pero en moi poucos casos totalmente oposta. Se ben só un único titular se mostrou totalmente remiso a explorar esta posibilidade (por ter feito un importante investimento en cerramentos e manter mala relación cos seus veciños), varios obstáculos potenciais foron identificados polos entrevistados:

a) As dificultades para manter superficies e usos que garantan o mantemento das axudas percibidas da Política Agrícola Común (PAC), particularmente tendo en conta o sistema de "rexións" existente na actualidade.

b) A falta de ganderías próximas coas que permutar, de modo que sería razoable implicar a titulares non dedicados a actividade agraria. Neste caso a competencia co uso forestal percíbese como un gran inconveniente.

c) As diferenzas no estado agronómico das parcelas. A maioría coincidía, non obstante, en que clasificar as parcelas en aptas ou non para o cultivo de millo sería unha simplificación aceptable.

d) A existencia de valados e árbores de lindeiros.

e) As relacións sociais co resto de participantes, especialmente á hora de permutar o uso de parcelas arrendadas.

f) A pouca marxe de mellora que queda nas parroquias onde os gandeiros xa foron permutando pola súa conta.

Respecto da entrevista cos dous técnicos de cooperativa, a motivación principal era coñecer a súa percepción acerca dos principais problemas da estrutura da base territorial das explotacións coas que traballan e o sentir xeral dos gandeiros aos que asesoran. Tamén exploramos as estratexias que levan ou pensan que poderían levarse a cabo para mellorar a estrutura das explotacións, así como polos principais escollos a salvar durante o procedemento, en especial orientado ó deseño da ferramenta de permuta de usos.

No relativo á estrutura das explotacións que forman parte da cooperativa na que traballan, a percepción xeral é que a maioría están afectadas por unha grande dispersión espacial e por un reducido tamaño medio das parcelas. Outro aspecto que destacan é a escasa superficie total que manexa cada explotación, debido fundamentalmente á existencia de moitas explotacións concentradas nun espazo relativamente reducido, pero tamén á escasa iniciativa por parte dos gandeiros, especialmente por parte daqueles que herdaron bases territoriais maiores ou mellor dimensionadas (que serían en moitos casos os que menos esforzos terían realizado para incrementar ou concentrar a superficie total que xestionan a través dalgunha combinación de compravenda e permuta). Ambos entrevistados coinciden en sinalar que a perspectiva (real 
ou imaxinada) dunha maior facilidade para acceder á terra no futuro ("xa haberá terra", "moitos pecharan e deixarán as terras", "non lles deixarán plantar e terán que arrendalas"...) parece motivar (ou xustificar) a referida falta de iniciativa.

No extremo contrario, algúns gandeiros terían sido particularmente activos na mellora da base territorial, ben porque partían dunha herdanza con poucas terras, porque tiñan máis iniciativa, por necesidades ineludibles (para ter acceso a axudas, para eliminar xurros...), ou ben por presentarse unha oportunidade (p.ex. porque coincidiu que se arrendaban ou vendían as parcelas lindeiras).

Con todo, o contexto actual de baixos prezos do leite, e o énfase na eficiencia na realización dos labores (a redución do seu custo horario, particularmente cando se contratan a terceiros), incrementan a presión sobre os gandeiros para explorar outras alternativas de xestión territorial.

A opinión dos técnicos dá menos importancia á distancia ata as parcelas da que manifestaron a maioría dos gandeiros, que en xeral parecían máis remisos a asumir grandes desprazamentos. En todo caso, en liña co manifestado por estes últimos, identificouse a superficie, acceso e tipo de manexo da explotación como aspectos relevantes á hora de decidir se unha distancia é asumible ou non, aos que se sumaría a existencia dunha fonte de materia orgánica próxima (p.ex. unha explotación avícola ou de gando porcino).

Sobre a viabilidade de realizar procesos de permuta de uso entre múltiples explotacións, das entrevistas deducimos que as permutas de propiedade son unha estratexia habitual das explotación máis activas, se ben o normal é que se realicen entre dúas explotacións e non en grupos máis amplos. Igual que sucedía nas entrevistas con gandeiros, a variación nas axudas percibidas da PAC aparece como un dos obstáculos máis importantes para a permuta de usos.

\section{Discusión}

En liña co descrito na bibliografía (Segers et al., 2010; Hiironen e Niukkanen, 2014), as entrevistas realizadas neste traballo permiten confirmar que a permuta de uso ou de propiedade entre diferentes gandeiros é unha práctica social habitual, como parte das estratexias adoptadas para mellorar a base territorial das súas explotacións. $\mathrm{Na}$ práctica, a permuta é unha ferramenta máis que se combina coa compra e o arrendamento en función das necesidades e das posibilidades de cada explotación e das oportunidades que o mercado de terras ofrece en cada momento (Vranken e Swinnen, 2006; Sklenicka et al., 2014).

Os límites e as posibilidades da permuta de terras están relacionados con institucións formais e informais (North, 1990; Ostrom, 1990) que condicionan as decisións individuais e regulan as regras ou condutas que se socializan nun grupo determinado (Ahlstrom and Bruton, 2002). As institucións deben ser tidas en consideración unha vez que guían comportamentos, percepcións e interaccións entre os actores sociais. A existencia de contratos verbais, por exemplo, indica a existencia de institucións informais e lazos sociais que anteceden á práctica da permuta. Por outro lado, os arranxos institucionais formais (regras específicas que os axentes establecen para as súas transaccións económicas ou nas relacións político-sociais e que definen como se coordinan os procedementos en campos específicos; Pires e Gomide, 2014) como as interaccións coas cooperativas ou as normas e regras relacionadas coa Política Agraria Común (PAC), condicionan o escenario de decisión individual e colectiva. Un exemplo desta afirmación é verificable polas reticencias ás permutas manifestado por algúns gandeiros ante risco de perder dereitos da PAC nunha rexión concreta (existen en Galicia 7 rexións diferenciadas de acordo cos R.D. 1076/2014, R.D. 1075/2014 e Regulamento (UE) $n^{\circ}$ 10307/2013 do Parlamento Europeo). En contraposición, outros non perciben isto como un problema sempre que non haxa cambio de uso nas parcelas afectadas e sempre as persoas que declaraban esas parcelas o seguiran facendo, o que tamén entendemos como un exemplo de arranxo informal para evitar limitacións establecidas polas institucións formais.

O feito de que as permutas de uso formen parte do conxunto de ferramentas utilizadas polos gandeiros no proceso de mellora da súa estrutura territorial permite supoñer que un instrumento lixeiramente diferente, consistente en ampliar o proceso de permuta a múltiples parcelas de múltiples titulares de cada vez, pode ser ben acollido. Malia os diferentes niveis de entusiasmo na recepción da idea, observamos que superadas as primeiras reticencias, os entrevistados aceptaban enseguida o debate arredor das súas potencialidades e dificultades en termos de posibilidade real. Entre as condicións necesarias para o éxito do proceso aparecen como salientables a necesidade de valorar a calidade agronómica das parcelas (p.ex. cun esquema simple de aptitude ou non para o cultivo de millo), ou o obxectivo de achegar as parcelas resultantes da permuta á explotación. En todo caso, probablemente non tódalas parcelas utilizadas por unha explotación nun momento concreto serían susceptibles de ser introducidas no proceso de permuta de uso, dadas as diferentes situacións da propiedade nas que poden atoparse: por exemplo, non sempre será posible permutar o uso de parcelas arrendadas. Por outra parte, restrinxir a participación a gandeiros ou agricultores en activo pode limitar considerablemente as posibilidades de mellora da estrutura pola vía da permuta en áreas onde son poucas as explotacións que manteñen a súa actividade. Desde este punto de vista, é evidente que a permuta debe verse como unha ferramenta máis que debe combinarse con outros instrumentos de xestión de terras e de negociación entre propietarios e xestores da terra para lograr os obxectivos que se marquen.

\section{Conclusións}

Podemos concluír que a viabilidade dun proceso de permutas de uso como o explorado neste traballo non depende tanto da capacidade técnica de proporcionar solucións de permuta (a través da ferramenta informática da que se dispoña) como de aspectos institucionais e sociais. 
Así, a predisposición á participación de propietarios non gandeiros ou non agricultores (moi condicionada a súa vez pola ordenación territorial e a regulación da competencia entre usos do solo), as experiencias previas dos participantes en procesos similares, ou a dispersión das explotacións nunha determinada área xeográfica, por exemplo, serán esenciais á hora de acadar melloras significativas na base territorial actual das explotacións. Os labores de mediación e apoio das institucións formais resultarían cruciais en caso de desexar que un instrumento deste tipo poida aplicarse de forma máis ou menos habitual en diferentes partes do territorio.

En canto ás restricións ou condicionantes para o deseño dunha ferramenta informática, as principais recomendacións céntranse en obter pezas da maior dimensión posible no menor radio de distancia posible, permitir introducir no cálculo unha valoración agronómica simplificada das parcelas, ou valorar a existencia de parcelas que non entran no proceso de permuta pero que o titular da explotación desexaría agrupar coas restantes.

Agradecementos Este traballo foi realizado no contexto do Grupo Operativo de Innovación "Deseño dunha aplicación informática para a mellora da xestión de terras nas cooperativas gandeiras" (Feader 2016/50B), concedido ao abeiro das axudas para a execución de grupos operativos da Asociación Europea de Innovación do ano 2016.

Os autores agradecen a boa disposición de tódalas persoas entrevistadas, así como aos dous revisores anónimos e a Dra. Nathália T. Cosmo da Silva polos seus comentarios. Por suposto, calquera erro existente é enteiramente responsabilidade dos autores.

\section{Bibliografía}

Abubakari, Z., van der Molen, P., Bennett, R., Kuusaana, E., 2016. Land consolidation, customary lands, and Ghana's northern savannah ecological zone: An evaluation of the possibilities and pitfalls. Land Use Policy 54, 386-398.

Aerts, J. C. J. H., van Herwijnen, M., Janssen, R., Stewart, T. J., 2005. Evaluating spatial design techniques for solving land-use allocation problems. Journal of Environmental Planning and Management 48 (1), 121-142.

Ahlstrom, D., \& Bruton, G. D., 2002. An institutional perspective on the role culture in shaping strategic actions by technology-focused entrepreneurial firms in China. Entrepreneurship: Theory and Practice, 26(4), 53-70.

Akkus, M., Karagoz, O., Dulger, O., July 2012. Automated land reallotment using genetic algorithm. In: International Symposium on Innovations in Intelligent Systems and Applications (INISTA), 1-5.

Balling, R. J., Taber, J. T., Brown, M. R., Day, K., 1999. Multiobjective urban planning using genetic algorithm. Journal of Urban Planning and Development 125 (2), 1699.

Bernal, C., 2000. Metodología de la investigación para administración y economía. Bogotá: Pearson Educación.
Binns, B., 1950. The consolidation of fragmented agricultural holdings. Food and Agriculture Organization of the United Nations.

Borgwardt, S., Brieden, A., Gritzmann, P., 2014. Geometric clustering for the consolidation of farmland and woodland. The Mathematical Intelligencer 36 (2), 37-44.

Cay, T., Iscan, F., 2011. Fuzzy expert system for land reallocation in land consolidation. Expert Systems with Applications 38 (9), 11055-11071.

Crecente, R., Alvarez, C., Fra, U., 2002. Economic, social and environmental impact of land consolidation in Galicia. Land Use Policy 19 (2), 135-147.

Deininger, K., Savastano, S., Carletto, C., 2012. Land Fragmentation, Cropland Abandonment, and Land Market Operation in Albania. World Development 40 (10), 21082122.

Demetriou, D., Stillwell, J., See, L., 2012. Land consolidation in Cyprus: Why is an Integrated Planning and Decision Support System required? Land Use Policy 29 (1), 131142.

FAO, 2003. The design of land consolidation pilot projects in Central and Eastern Europe. Technical Report 6, United Nations Food and Agriculture Organization.

Guanghui, J., Xinpan, W., Wenju, Y., Ruijuan, Z., 2015. A new system will lead to an optimal path of land consolidation spatial management in China. Land Use Policy 42, 27-37.

Haldrup, N. O., 2015. Agreement based land consolidation in perspective of new modes of governance. Land Use Policy 46, 163-177.

Hartvigsen, M., 2014. Land reform and land fragmentation in Central and Eastern Europe. Land Use Policy 36 (0), 330341.

Hiironen, J., Niukkanen, K., 2014. On the structural development of arable land in Finland - How costly will it be for the climate? Land Use Policy 36 (0), 192-198.

Implications for the Roles of State and Community in PostSocialist Land Consolidation. World Development 37 (8), 1411-1423.

Kai, C., Bo, H., Qing, Z., Shengxiao, W., 2009. Land use allocation optimization towards sustainable development based on genetic algorithm. In: 17th International Conference on Geoinformatics, pp. 1-5.

Kilgore, M. A., Snyder, S. A., 2016. Exploring the relationship between parcelization metrics and natural resource managers' perceptions of forest land parcelization intensity. Landscape and Urban Planning 149, 43-48.

Kupidura, A., Luczewski, M., Home, R., Kupidura, P., 2014. Public perceptions of rural landscapes in land consolidation procedures in Poland. Land Use Policy 39 (0), 313-319. 
Latruffe, L., Piet, L., 2014. Does land fragmentation affect farm performance? A case study from Brittany, France. Agricultural Systems 129 (0), 68-80.

Lisec, A., Primozic, T., Ferlan, M., Sumrada, R., Drobne, S., 2014. Landowners' perception of land consolidation and their satisfaction with the results - Slovenian experiences. Land Use Policy 38 (0), 550-563.

Liu, Y., Tang, W., He, J., Liu, Y., Ai, T., Liu, D., 2015. A landuse spatial optimization model based on genetic optimization and game theory. Computers, Environment and Urban Systems 49, 1-14.

Malhotra, N. K., 1997. Investigación de mercados. Pearson Educación.

man Hui, E. C., ping Leung, B. Y., hung Yu, K., 2014. The impact of different land-supplying channels on the supply of housing. Land Use Policy 39 (0), 244-253.

Manjunatha, A., Anik, A. R., Speelman, S., Nuppenau, E., 2013. Impact of land fragmentation, farm size, land ownership and crop diversity on profit and efficiency of irrigated farms in India. Land Use Policy 31 (0), 397-405.

Martínez, R., Solla, M., Arias, P., Armesto, J., 2013. Semiautomatic land consolidation software based on geographic information systems. Computers and Electronics in Agriculture 97 (0), 1-5.

Muchová, Z., Leitmanová, M., Petrovic, F., 2016. Possibilities of optimal land use as a consequence of lessons learned from land consolidation projects (Slovakia). Ecological Engineering 90, 294-306.

North, D. C., 1990. Institutions, institutional change and economic performance. Cambridge university press.

Orea, L., Perez, J. A., Roibas, D., 2015. Evaluating the double effect of land fragmentation on technology choice and dairy farm productivity: A latent class model approach. Land Use Policy 45 (0), 189-198.

Ostrom, E., 1990. Governing the commons. Cambridge university press

Pasakarnis, G., Maliene, V., 2010. Towards sustainable rural development in Central and Eastern Europe: Applying land consolidation. Land Use Policy 27 (2), 545-549.

Pasakarnis, G., Morley, D., Maliene, V., 2013. Rural development and challenges establishing sustainable land use in Eastern European countries. Land Use Policy 30 (1), 703-710.

Pires, R. R. C., \& Gomide, A. D. A., 2014. Burocracia, democracia e políticas públicas: arranjos institucionais de políticas de desenvolvimento (No. 1940). Texto para Discussão, Instituto de Pesquisa Econômica Aplicada (IPEA).

Porta, J., Parapar, J., Doallo, R., Barbosa, V., Santé, I., Crecente, R., Díaz, C., 2013a. A population-based iterated greedy algorithm for the delimitation and zoning of rural settlements. Computers, Environment and Urban Systems $39(0), 12-26$.
Porta, J., Parapar, J., Doallo, R., Rivera, F. F., Santé, I., Crecente, R., 2013b. High performance genetic algorithm for land use planning. Computers, Environment and Urban Systems 37 (0), 45-58.

Rahman, S., Rahman, M., 2009. Impact of land fragmentation and resource ownership on productivity and efficiency: The case of rice producers in bangladesh. Land Use Policy 26 (1), 95-103.

Real Decreto 1075/2014, de 19 de diciembre, sobre la aplicación a partir de 2015 de los pagos directos a la agricultura y a la ganadería y otros regímenes de ayuda, así como sobre la gestión y control de los pagos directos y de los pagos al desarrollo rural. Dispoñible en: https://www.boe.es/diario boe/txt.php?id=BOE-A-201413256.

Real Decreto 1076/2014, de 19 de diciembre, sobre asignación de derechos de régimen de pago básico de la Política Agrícola Común https://www.boe.es/diario boe/txt.php?id=BOE-A-201413257

Reglamento (UE) № 1307/2013, de 17 de diciembre, por el que se establecen normas aplicables a los pagos directos a los agricultores en virtud de los regímenes de ayuda incluidos en el marco de la Política Agraria Común y por el que se derogan los Reglamentos (CE) n 637/2008 y (CE) $n^{\circ} 73 / 2009$ del Consejo. Dispoñible en: https://www.boe.es/doue/2013/347/L00608-00670.pdf

Rendenieks, Z., gerts Nikodemus, O., Br umelis, G., 2015. The implications of stand composition, age and spatial patterns of forest regions with different ownership type for management optimisation in northern Latvia. Forest Ecology and Management 335, 216-224.

Schaich, H., Plieninger, T., 2013. Land ownership drives stand structure and carbon storage of deciduous temperate forests. Forest Ecology and Management 305, 146-157.

Segers, K., Dessein, J., Hagberg, S., Teklebirhan, Y., Haile, M., Deckers, J., 2010. Unravelling the dynamics of access to farmland in Tigray, Ethiopia: The 'emerging land market' revisited. Land Use Policy 27 (4), 1018-1026.

Sikor, T., Müller, D., 2009. The Limits of State-Led Land Reform: An Introduction. World Development 37 (8), 13071316.

Sikor, T., Müller, D., Stahl, J., 2009. Land Fragmentation and Cropland Abandonment in Albania:

Sklenicka, P., Janovska, V., Salek, M., Vlasak, J., Molnarova, K., 2014. The farmland rental paradox: Extreme land ownership fragmentation as a new form of land degradation. Land Use Policy 38 (0), 587-593.

Stewart, T. J., Janssen, R., 2014. A multiobjective gis-based land use planning algorithm. Computers, Environment and Urban Systems 46, 25-34.

Stewart, T. J., Janssen, R., Herwijnen, M., 2004. A genetic algorithm approach to multiobjective land use planning. Computers \& Operations Research 31, 2293-2313. 
Suárez, M., Santé, I., Rivera, F. F., Crecente, R., Boullón, M., Porta, J., Parapar, J., Doallo, R., 2011. A Parallel Algorithm Based On Simulated Annealing For Land Use Zoning Plans. In: International Conference on Parallel and Distributed Processing Techniques and Applications (PDPTA). Las Vegas, Nevada, EEUU, pp. 360-366.

Uyan, M., Cay, T., Inceyol, Y., Hakli, H., 2015. Comparison of designed different land reallocation models in land consolidation: A case study in Konya/Turkey. Computers and Electronics in Agriculture 110 (0), 249-258.

Vallejo, M., Rieser, V., Corne, D. W., 2015. Genetic algorithm evaluation of green search allocation policies in multilevel complex urban scenarios. Journal of Computational Science 9, 57-63.

van Dijk, T., 2003. Scenarios of Central European land fragmentation. Land Use Policy 20 (2), 149-158.

van Dijk, T., Kopeva, D., 2006. Land banking and Central Europe: future relevance, current initiatives, Western European past experience. Land Use Policy 23 (3), 286301.
Vranken, L., Swinnen, J., 2006. Land rental markets in transition: Theory and evidence from Hungary. World Development 34 (3), 481-500.

Xibao, X., Jianming, Z., Xiaojian, Z., 2006. Integrating GIS, cellular automata and genetic algorithm in urban spatial optimization. A case study of Lanzhou. In: Proc of SPIE. Vol. 6420. 64201U-1 - 64201U-10.

Xin, H., Zhi-xia, Z., 2008. Application of genetic algorithm to spatial distribution in urban planning. In: IEEE International Symposium on Knowledge Acquisition and Modeling Workshop. pp. 1026-1029, wuhan, China.

Yan, J., Xia, F., Bao, H. X., 2015. Strategic planning framework for land consolidation in China: A top-level design based on SWOT analysis. Habitat International 48, 46-54.

Zhang, H. H., Zeng, Y. N., Bian, L., 2010. Simulating multiobjective spatial optimization allocation of land use based on the integration of multi-agent system and genetic algorithm. International Journal of Environmental Research 4 (4), 765-776. 